\title{
Soroprevalência e fatores associados a infecções crônicas entre idosos residentes na comunidade
}

\author{
Seroprevalence and factors associated with chronic infections \\ among community-dwelling elderly individuals
}

Sérgio Viana Peixoto (https://orcid.org/0000-0001-9431-2280) 1,2

Karen Cecília Lima Torres (https://orcid.org/0000-0002-2943-4350) ${ }^{3}$

Andréa Teixeira-Carvalho (https://orcid.org/0000-0003-2814-5183) ${ }^{3}$

Olindo Assis Martins-Filho (https://orcid.org/0000-0002-5494-4889) ${ }^{3}$

Maria Fernanda Lima-Costa (https://orcid.org/0000-0002-3474-2980)

${ }^{1}$ Núcleo de Estudos em Saúde Pública e Envelhecimento, Instituto René Rachou, Fiocruz Minas. Av. Augusto de Lima 1715, Barro Preto. 30190-009 Belo Horizonte MG Brasil. sergio.peixoto@fiocruz.br ${ }^{2}$ Departamento de Gestão em Saúde, Escola de

Enfermagem, Universidade Federal de Minas Gerais. Belo Horizonte MG Brasil.

${ }^{3}$ Grupo Integrado de Pesquisas em Biomarcadores, Instituto René Rachou, Fiocruz Minas. Belo Horizonte MG Brasil.
Abstract Chronic infections can contribute to the aging process, but this issue is less studied in Latin America. The aim was to assess the prevalence and factors associated with cytomegalovirus (CMV), Herpes Simplex 1 (HSV-1), Chlamydia pneumoniae and Helicobacter pylori among the elderly. A total of 1,320 individuals participated from the baseline of the Elderly Cohort of Bambui. IgG antibodies against infections and explanatory variables (sociodemographic factors, health behaviors and health conditions) were evaluated. Poisson regression models with robust variance were used. Seroprevalence rates were $99.4 \%$ for CMV, 96.7\% for HSV-1, 56\% for C. pneumoniae and $70.5 \%$ for $\mathrm{H}$. pylori. Elderly men, women, smoke$r s$, diabetics, the disabled and those with high levels of IL-6 had a higher prevalence of CMV. $H S V-1$ was less frequent among women. The prevalence of $C$. pneumoniae was higher at ages $>75$ and among diabetics; it was lower among women and individuals with less schooling. H. pylori was less frequent among women and those with detectable levels of $I L-1 \beta$, but more common among smokers. The findings show a high prevalence of chronic infection and a different epidemiologic profile for each pathogen, making it possible to detect groups that are vulnerable to these infections. Key words Communicable diseases, Health of the Elderly, Health Surveys, Epidemiologic factors
Resumo Infecções crônicas podem contribuir com o processo de envelhecimento, mas isso ainda é pouco explorado na América Latina. O objetivo foi avaliar a prevalência e os fatores associados ao citomegalovírus (CMV), Herpes simples 1 (HSV-1), Chlamydia pneumoniae e Helicobacter pylori entre idosos. Participaram 1.320 indivíduos da linha de base da Coorte de Idosos de Bambuí. Foram avaliados anticorpos ( $I g G)$ para as infecções e variáveis exploratórias (sociodemográficas, comportamentos em saúde e condições de saúde). Utilizaram-se modelos de regressão de Poisson com variância robusta. A prevalência foi de 99,4\% para CMV, 96,7\% para $H S V-1,56,0 \%$ para C. pneumoniae e $70,5 \%$ para $H$. pylori. Os mais velhos, mulheres, fumantes, diabéticos, incapazes e com maiores niveis de IL-6 tinham maior prevalência de CMV. HSV-1 foi menos frequente entre as mulheres. Infecção por C. pneumoniae foi maior entre os mais velhos e diabéticos; e menor entre mulheres e os menos escolarizados. H. pylori foi menos frequente entre as mulheres e naqueles com maiores niveis de IL-1ß, mas mais comuns entre os fumantes. Os achados mostram elevada prevalência de infecções crônicas e diferentes perfis epidemiológicos para cada patógeno, permitindo a detecção de grupos vulneráveis a essas infecções. Palavras-chave Doenças Transmissiveis, Saúde do Idoso, Inquéritos Epidemiológicos, Fatores Epidemiológicos 


\section{Introdução}

O estudo dos fatores associados ao processo de envelhecimento é de extrema importância para a saúde pública, considerando o crescente aumento da população idosa observado em todo o mundo. Nesse sentido, tem sido proposto que infecções por alguns patógenos, como cytomegalovírus (CMV), herpes simples (HSV), Clamydia pneumoniae e Helicobacter pylori, podem desempenhar importante papel nesse processo, em função das suas associações com doença cardiovascular, diabetes, declínio cognitivo, fragilidade e até mortalidade ${ }^{1-4}$. Chama atenção que, de forma semelhante a outros agravos à saúde, as infecções crônicas podem ser mais frequentes e de maior gravidade entre idosos, o que pode explicar seu efeito prejudicial nessa população ${ }^{5}$.

Prevalências dos herpesvírus, como CMV e HSV-1, atingem valores superiores a $60 \%$ na faixa etária de 50 anos ou mais, sobretudo nas regiões menos desenvolvidas, podendo chegar a $90 \%$ ou mais em algumas populações ${ }^{6,7}$. Por sua vez, a prevalência da infecção por $C$. pneumoniae apresenta ampla variação na literatura, atingindo $75 \%$ na população adulta italiana ${ }^{8}$, de forma semelhante ao observado para $H$. pylori que chega a $63,4 \%$ na América Latina e Caribe e $79,1 \%$ na África9.

Alguns estudos apontam que essas infecções tendem a ser mais comuns entre os mais velhos, homens (com exceção do CMV), aqueles com pior condição socioeconômica, piores comportamentos em saúde e com doenças crônicas, sobretudo as cardiovasculares ${ }^{1,6,9-14}$. No entanto, $\mathrm{O}$ perfil epidemiológico é muito variável entre os patógenos e entre as populações, não sendo consistente em diferentes países.

De maneira geral, as infecções crônicas apresentam-se associadas a eventos cardiovasculares $^{5,15}$ e declínio físico e cognitivo ${ }^{1,14}$, embora esses achados não tenham sido reproduzidos em algumas populações ${ }^{2,3,16}$. Entre os possíveis mecanismos que explicam a associação entre infecções crônicas e piores condições de saúde destaca-se a amplificação ou perpetuação de um estado pró-inflamatório na presença dessas infecções, contribuindo para o processo de imunosenescência ${ }^{1,5,17}$ e agravando as condições de saúde dos idosos.

Portanto, apesar de alguns resultados controversos na literatura, as infecções crônicas parecem exercer importante influência no processo de envelhecimento, tanto pelo dano direto causado pelo patógeno nos tecidos infectados, quanto pelo impacto indireto no organismo, sobretudo pelo processo inflamatório, mecanismo conhecido como inflamm-aging ${ }^{18}$. Ressalta-se que a maioria dos estudos são conduzidos em países desenvolvidos e em populações adultas, sendo essas associações pouco exploradas entre idosos residentes em regiões de baixa e média renda, nas quais esse quadro de infecções pode ser mais grave ${ }^{17}$.

O objetivo do presente trabalho é avaliar a prevalência e os fatores associados a quatro infecções crônicas (CMV, HSV-1, C. pneumoniae e H. pylori) entre idosos residentes na comunidade. Pelo nosso conhecimento, essa constitui a primeira proposta para o estudo sobre o perfil das infecções crônicas em uma população idosa residente em um país latino-americano, o que poderá contribuir para um melhor entendimento desse aspecto em nosso meio.

\section{Metodologia}

\section{Desenho, área e população de estudo}

A Coorte de Idosos de Bambuí é conduzida na cidade de mesmo nome (cerca de 15.000 habitantes), situada em Minas Gerais. A linha de base da coorte foi constituída em 1997, quando toda população com 60 anos ou mais $(\mathrm{n}=1.742)$, residente na cidade, foi convidada a participar da pesquisa. O delineamento da coorte e os procedimentos adotados foram descritos com maiores detalhes em publicação anterior ${ }^{19}$. Para o presente estudo foram considerados todos os idosos participantes da linha de base que tiveram as dosagens de anticorpos para as infecções de interesse e possuíam informações completas para todas as demais variáveis incluídas na análise, totalizando $1.320(75,8 \%)$ participantes.

O Estudo de Coorte de Idosos de Bambuí foi aprovado pelo Comitê de Ética em Pesquisa da Fundação Oswaldo Cruz e cada participante assinou um termo de consentimento livre e esclarecido para participarem da pesquisa.

\section{Desfechos estudados: infecções crônicas}

Foram consideradas as infecções por herpes simples tipo 1 (HSV-1), citomegalovírus (CMV), C. pneumoniae e H. pylori, pela avaliação de anticorpos (IgG) no soro dos participantes, coletado na linha de base da coorte e armazenado a $-80^{\circ} \mathrm{C}$; as dosagens foram realizadas em 2018 . O ensaio de imunoabsorção enzimática (ELISA) quantitativo foi usado para avaliação dos anticorpos 
para HSV-1, C. pneumoniae e H. pylori, utizando kits da Euroimmun (Alemanha) para os dois primeiros patógenos e o kit SERION ELISA classic (Alemanha) para H. pylori. Já os anticorpos IgG anti-citomegalovírus foram avaliados pelo ensaio de eletroquimioluminescência, utilizando o kit Elecsys (Roche Diagnostics, Suíça). Todos os testes foram realizados seguindo as orientações dos fabricantes dos kits, utilizando procedimentos padronizados e analisadores automatizados, em laboratórios de referência e com experiência nesse tipo de dosagem.

De acordo com as orientações dos fabricantes, os testes foram considerados positivos para o patógeno utilizando-se os seguintes pontos de corte: HSV-1 ( $\geq 22 \mathrm{UR} / \mathrm{mL}), \mathrm{CMV}(\geq 1,00 \mathrm{U} / \mathrm{mL})$, C. pneumoniae ( $\geq 22 \mathrm{UR} / \mathrm{mL})$ e H. pylori $(>50 \mathrm{U} /$ $\mathrm{mL}$ ). Para a presente análise, devido à elevada positividade para HSV-1 e CMV e considerando a influência do nível de anticorpos dessas infecções em diversos eventos adversos ${ }^{4,17}$, os desfechos foram definidos pela titulação acima da mediana (>160 UR/mL para HSV-1 e >399,5 U/mL para CMV).

\section{Variáveis explicativas}

As variáveis explicativas foram selecionadas baseando-se em resultados prévios sobre os fatores associados a infecções crônicas ${ }^{1-4}$ e incluíram características sociodemográficas, comportamentais e relacionadas às condições de saúde. Entre os fatores sociodemográficos foram consideradas a idade em anos (60 a 74 e 75 ou mais), o sexo (homens, mulheres) e a escolaridade em anos de estudo (4 ou mais e 0 a 3 anos). Os comportamentos em saúde incluíram tabagismo atual ( sim, não) e consumo de álcool ( $<7$ e 7 ou mais doses por semana $)^{20}$. Os tabagistas atuais eram os idosos que reportaram já ter consumido 100 ou mais cigarros ao longo da vida e continuavam fumando no momento da entrevista. O consumo de álcool foi avaliado pela informação sobre o número de dias por semana que fazia uso de álcool e o número de doses por dia, considerando o consumo habitual. Uma dose de bebida alcoólica era referente a uma lata de cerveja, uma taça de vinho ou uma dose de bebida destilada.

As variáveis relacionadas às condições de saúde incluíram diabetes, hipertensão arterial, doença cardiovascular, déficit cognitivo e incapacidade, além de quatro marcadores inflamatórios associados a infecções crônicas e ao envelhecimento [interleucina 6 (IL-6), proteína C-reativa (PCR), interleucina 1 beta (IL-1 $\beta$ ) e fator de necrose tumoral (TNF) $]^{1-4}$. O diagnóstico de diabetes foi definido pelo nível de glicemia de jejum maior ou igual a $126 \mathrm{mg} / \mathrm{dL}$ e/ou uso de medicamentos (insulina ou hipoglicemiantes orais). A hipertensão arterial foi definida por alterações nos valores da pressão arterial (pressão sistólica maior ou igual a $140 \mathrm{mmHg}$ e/ou pressão diastólica maior ou igual a $90 \mathrm{mmHg}$ ) e/ou pelo uso de medicamentos. A presença de doença cardiovascular foi avaliada pela existência de pelo menos uma entre três condições (acidente vascular encefálico - AVE, infarto do miocárdio e angina). A história de $\mathrm{AVE}^{21}$ e presença de angina ${ }^{22}$ foram verificadas pela utilização de instrumentos padronizados, e o infarto foi avaliado pela história de diagnóstico médico para essa condição. $\mathrm{O}$ comprometimento cognitivo foi avaliado pela pontuação inferior a 22 da versão brasileira do questionário Mini-Mental State Examination (MMSE), correspondente ao quartil inferior, que se mostrou adequado à população idosa de Bambuín ${ }^{23}$. A limitação funcional foi avaliada pelo desempenho em executar atividades básicas e instrumentais de vida diária, incluindo tomar banho, vestir roupa, alimentar sozinho, realizar transferência, administrar o próprio dinheiro, realizar trabalhos domésticos e preparar o próprio alimento. A presença de limitação foi definida como a impossibilidade em executar pelo menos uma dessas atividades sem ajuda de outra pessoa.

As dosagens das citocinas foram realizadas no soro dos participantes, por um ensaio de imunofluorescência (método de Cytometric Bead Array - CBA), utilizando-se anticorpos anti-citocinas marcados com ficoeritrina para indicar a intensidade média de fluorescência (MFI). Os dados de MFI foram obtidos usando citômetro de fluxo FACSVerse flow cytometer (Becton Dickinson, Estados Unidos) e os resultados foram expressos em $\mathrm{pg} / \mathrm{mL}$, calculados pelo software BD FCAP Array 3.0 (Becton Dickinson, Estados Unidos). A dosagem da PCR ultra-sensível foi realizada pelo método imunonefelométrico em um nefelométrico automático (BNII ${ }^{\mathrm{TM}}$, Dade Behring, Alemanha) e expressa em mg/dL. As dosagens de IL-6 e PCR foram dicotomizadas no percentil 75 da distribuição (altos produtores) e os níveis de IL1- $\beta$ e TNF foram divididos em detectáveis e não detectáveis.

As informações foram coletadas por meio de entrevistas, exame físico e coleta de sangue. As entrevistas foram realizadas na residência do participante e respondidas pelo próprio idoso, exceto quando estava impossibilitado por problema de saúde, utilizando-se nesse caso um respondente substituto. $\mathrm{O}$ uso de medicamentos 
foi verificado nas entrevistas, considerando todos os medicamentos usados nos 90 dias anteriores à entrevista, pela conferência da embalagem ou receita médica. A coleta de sangue e as medidas de pressão arterial foram realizadas na clínica de campo do projeto, ou no domicílio do participante, quando havia impossibilidade de locomoção do idoso. A determinação da pressão arterial foi realizada utilizando-se um esfignomanômetro de mercúrio, tendo sido realizadas três medidas, considerando-se a média da segunda e da terceira medidas. As medidas de pressão foram realizadas com o indivíduo sentado, após $5 \mathrm{mi}-$ nutos de repouso e pelo menos 30 minutos sem ingestão de cafeína e/ou uso de cigarros. Os níveis de glicemia no sangue foram determinados utilizando-se kits comerciais (Boehringer Mannhein, Alemanha) e analisador automático.

\section{Análise dos dados}

Foi realizada a descrição da prevalência de maiores títulos de anticorpos para CMV e HSV1 , além da prevalência de positividade para $C$. pneumoniae e $H$. pylori para a população geral e segundo as variáveis exploratórias consideradas. As associações não ajustadas entre cada infecção e as variáveis estudadas foram avaliadas pelo teste do qui-quadrado de Pearson.

Posteriormente, foram construídos modelos não ajustados, pela regressão de Poisson com variância robusta, para avaliar a magnitude da associação entre as infecções e cada variável exploratória, obtendo-se os valores de razões de prevalência (RP) e respectivos intervalos de confiança com 95\% (IC95\%).

Por fim, foram construídos modelos múltiplos, para cada infecção pesquisada, seguindo um ajuste hierarquizado: os primeiros modelos incluíram as variáveis sociodemográficas e comportamentais, produzindo-se os valores de RP e IC (95\%) ajustado por essas condições; os demais modelos incluíram todas as condições relacionadas à saúde consideradas nesse estudo, além do ajuste pelas condições sociodemográficas e comportamentais.

Além disso, foi realizada uma análise de sensibilidade para verificar o impacto da exclusão dos indivíduos com resultados inconclusivos para dosagens de anticorpos, segundo orientações do fabricante. Entre os idosos incluídos no presente estudo, $0,2 \%$ tinham resultados inconclusivos para CMV $(0,50$ a $0,99 \mathrm{U} / \mathrm{mL}), 0,3 \%$ para HSV-1 (16 a $21 \mathrm{UR} / \mathrm{mL}$ ), 8,7\% para C. pneumoniae (16 a $21 \mathrm{UR} / \mathrm{mL}$ ) e $4,4 \%$ para $H$. pylori ( 35 a $50 \mathrm{U} / \mathrm{mL}$ ).

\section{Resultados}

A prevalência de sorologia positiva foi igual a 99,4\% (IC95\%: 98,8-99,7\%) para citomegalovírus, 96,7\% (IC95\%: 95,6-97,6) para herpes simples tipo 1, 56,0\% (IC95\%: 53,3-58,6) para $C$. pneumoniae e 70,5\% (IC95\%: 68,0-72,9) para $H$. pylori.

A Tabela 1 descreve a prevalência das infecções investigadas, segundo as variáveis exploratórias pesquisadas. A prevalência de maiores títulos de anticorpos para CMV foi maior entre as mulheres, entre os idosos que consumiam menor quantidade de bebidas alcoólicas, que tinham diagnóstico de diabetes e que reportavam limitações em atividades de vida diária e entre aqueles com maiores dosagens de IL-6 e PCR. Para o HSV-1, apenas a variável sexo mostrou associação significativa na análise não ajustada, sendo a maior prevalência de títulos elevados de anticorpos para esse vírus observada entre os homens. Já a infecção por C. pneumoniae foi maior entre os mais velhos, os homens, aqueles com maior escolaridade, os fumantes atuais e entre os idosos com diagnóstico de diabetes. Por fim, a prevalência de infecção por $H$. pylori foi maior entre os homens e entre os fumantes atuais; por outro lado, essa infecção foi mais comum entre os idosos que não relataram limitações em atividades de vida diária e entre aqueles com níveis não detectáveis de IL-1 $\beta$.

A Tabela 2 descreve a magnitude das associações entre as infecções crônicas estudadas e as variáveis exploratórias incluídas nesse estudo. Todas as associações significativas descritas na tabela anterior podem também ser observadas nessa tabela, que mostra as razões de prevalência e respectivos intervalos de confiança (95\%).

A associação independente entre as infecções crônicas e as variáveis sociodemográficas e comportamentais estão descritas na Tabela 3. Após ajustamento por todas as variáveis listadas na tabela, a maior titulação de anticorpos para CMV foi mais prevalente entre os idosos mais velhos (Razão de Prevalência - RP: 1,13; IC95\%: 1,011,28 ), as mulheres (RP: 1,46; IC95\%: 1,28-1,66) e os tabagistas atuais (RP: 1,17; IC95\%: 1,02-1,35). Por outro lado, maiores títulos de anticorpos para HSV-1 foram menos frequentes entre as mulheres (RP: 0,78; IC95\%: 0,70-0,88). A infecção por $C$. pneumoniae foi mais frequente entre os idosos mais velhos (RP: 1,15; IC95\%: 1,04$1,28)$ e menos frequente entre as mulheres (RP: 0,80; IC95\%: 0,73-0,89) e entre aqueles com menor escolaridade (RP: 0,86; IC95\%: 0,78-0,95). Já 
Tabela 1. Prevalência da soropositividade para citomegalovírus (CMV), herpes simples tipo 1 (HSV-1), C. pneumoniae (CP) e H. pylori (HP), segundo características sociodemográficas, comportamentais e de saúde. Linha de base da Coorte de idosos de Bambuí.

\begin{tabular}{|c|c|c|c|c|}
\hline \multirow{2}{*}{ Variáveis } & \multicolumn{4}{|c|}{ Prevalência - \% } \\
\hline & CMV & HSV & $\mathrm{CP}$ & HP \\
\hline \multicolumn{5}{|l|}{ Idade em anos } \\
\hline 60 a 74 & 48,5 & 49,7 & $54,4^{*}$ & 71,6 \\
\hline 75 e mais & 54,7 & 50,2 & 61,7 & 66,6 \\
\hline \multicolumn{5}{|l|}{ Sexo } \\
\hline Homens & $39,0^{*}$ & $57,7^{\star}$ & $64,4^{*}$ & $75,8^{*}$ \\
\hline Mulheres & 56,7 & 44,8 & 50,7 & 67,2 \\
\hline \multicolumn{5}{|l|}{ Escolaridade em anos } \\
\hline 4 ou mais & 48,7 & 50,6 & $61,3^{*}$ & 70,3 \\
\hline$<4$ & 50,5 & 49,3 & 53,0 & 70,7 \\
\hline \multicolumn{5}{|l|}{ Tabagismo atual } \\
\hline Não & 49,6 & 48,7 & $54,7^{\star}$ & $68,8^{*}$ \\
\hline Sim & 50,9 & 54,7 & 61,9 & 78,4 \\
\hline \multicolumn{5}{|c|}{ Consumo de álcool (doses/semana) } \\
\hline$<7$ & $50,8^{*}$ & 49,4 & 55,6 & 70,4 \\
\hline 7 ou mais & 31,8 & 56,1 & 65,2 & 72,7 \\
\hline \multicolumn{5}{|l|}{ Diabetes $^{1}$} \\
\hline Não & $48,5^{\star}$ & 49,1 & $54,5^{\star}$ & 70,8 \\
\hline Sim & 57,5 & 53,9 & 64,8 & 68,9 \\
\hline \multicolumn{5}{|l|}{ Hipertensão arterial $^{2}$} \\
\hline Não & 47,4 & 49,4 & 56,2 & 68,3 \\
\hline Sim & 51,4 & 50,0 & 55,9 & 71,9 \\
\hline \multicolumn{5}{|l|}{ Doença cardiovascular ${ }^{3}$} \\
\hline Não & 49,9 & 50,1 & 56,7 & 69,9 \\
\hline $\operatorname{Sim}$ & 49,5 & 48,1 & 52,4 & 73,8 \\
\hline \multicolumn{5}{|c|}{ Comprometimento cognitivo $^{4}$} \\
\hline Não & 49,8 & 48,5 & 56,4 & 70,7 \\
\hline Sim & 50,0 & 54,8 & 54,4 & 70,0 \\
\hline \multicolumn{5}{|l|}{ Limitação funcional $^{5}$} \\
\hline Não & $48,0^{*}$ & 50,3 & 55,4 & $71,7^{*}$ \\
\hline $\operatorname{Sim}$ & 60,7 & 46,9 & 59,2 & 63,8 \\
\hline \multicolumn{5}{|l|}{ Interleucina 6} \\
\hline$<1,08 \mathrm{pg} / \mathrm{mL}$ & $45,6^{*}$ & 47,7 & 53,5 & 72,4 \\
\hline$\geq 1,08 \mathrm{pg} / \mathrm{mL}$ & 54,1 & 51,8 & 58,5 & 68,6 \\
\hline \multicolumn{5}{|l|}{ Proteína C-reativa } \\
\hline$<3,25 \mathrm{mg} / \mathrm{dL}$ & $45,4^{*}$ & 49,2 & 55,2 & 72,5 \\
\hline$\geq 3,25 \mathrm{mg} / \mathrm{dL}$ & 54,3 & 50,4 & 56,8 & 68,6 \\
\hline \multicolumn{5}{|l|}{ Interleucina $1 \mathrm{~b}$} \\
\hline Não detectável & 50,3 & 50,1 & 56,3 & $72,4^{*}$ \\
\hline$\geq 0,01 \mathrm{pg} / \mathrm{mL}$ & 48,1 & 48,4 & 54,8 & 63,6 \\
\hline \multicolumn{5}{|c|}{ Fator de necrose tumoral - TNF } \\
\hline Não detectável & 50,1 & 50,0 & 55,7 & 70,8 \\
\hline$\geq 0,02 \mathrm{pg} / \mathrm{mL}$ & 48,7 & 48,7 & 57,6 & 69,2 \\
\hline
\end{tabular}

CMV: citomegalovírus (IgG>399,5 U/mL); HSV: herpes simples (IgG>160 UR/mL); CP: Chlamydia pneumoniae (IgG $>22$ UR/ $\mathrm{mL}$ ); HP: Helicobacter pylori (positivo - IgG $>50 \mathrm{U} / \mathrm{mL}$ ). ${ }^{1}$ Glicemia de jejum $>126 \mathrm{mg} / \mathrm{dL}$ e/ou uso de medicamento; ${ }^{2}$ Pressão sistólica $\geq 140 \mathrm{mmHg}$ e/ou pressão diastólica $\geq 90 \mathrm{mmHg}$ e/ou tratamento; ${ }^{3}$ Angina (protocolo), relato de infarto do miocárdio e/ou relato de acidente vascular encefálico; ${ }^{4}$ Escore do Mini-exame do estado mental $<22 ;{ }^{5}$ Relato de alguma dificuldade para tomar banho, vestir-se, alimentar-se, deitar e levantar da cama, lidar com finanças, fazer tarefas domésticas ou preparar refeições. ${ }^{*} \mathrm{p}<0,05$.

Fonte: Elaborado pelos autores. 
Tabela 2. Resultados da análise de associação (não ajustada) entre citomegalovírus (CMV), herpes simples tipo 1 (HSV-1), C. pneumoniae (CP) e H. pylori (HP) e características sociodemográficas, comportamentais e de saúde. Linha de base da Coorte de idosos de Bambuí.

\begin{tabular}{|c|c|c|c|c|}
\hline \multirow{2}{*}{ Variáveis } & \multicolumn{4}{|c|}{ RP bruta (IC95\%) } \\
\hline & CMV & HSV & $\mathrm{CP}$ & HP \\
\hline \multicolumn{5}{|l|}{ Idade em anos } \\
\hline 60 a 74 & 1,00 & 1,00 & 1,00 & 1,00 \\
\hline 75 e mais & $1,12(0,99-1,28)$ & $1,01(0,89-1,15)$ & $1,13(1,02-1,26)$ & $0,93(0,85-1,02)$ \\
\hline \multicolumn{5}{|l|}{ Sexo } \\
\hline Homens & 1,00 & 1,00 & 1,00 & 1,00 \\
\hline Mulheres & $1,45(1,28-1,65)$ & $0,78(0,70-0,86)$ & $0,79(0,72-0,87)$ & $0,89(0,83-0,95)$ \\
\hline \multicolumn{5}{|l|}{ Escolaridade em anos } \\
\hline 4 ou mais & 1,00 & 1,00 & 1,00 & 1,00 \\
\hline$<4$ & $1,04(0,92-1,16)$ & $0,97(0,87-1,09)$ & $0,86(0,79-0,95)$ & $1,01(0,94-1,08)$ \\
\hline \multicolumn{5}{|l|}{ Tabagismo atual } \\
\hline Não & 1,00 & 1,00 & 1,00 & 1,00 \\
\hline $\operatorname{Sim}$ & $1,02(0,89-1,18)$ & $1,12(0,98-1,28)$ & $1,13(1,01-1,27)$ & $1,14(1,05-1,23)$ \\
\hline \multicolumn{5}{|c|}{ Consumo de álcool (doses/semana) } \\
\hline$<7$ & 1,00 & 1,00 & 1,00 & 1,00 \\
\hline 7 ou mais & $0,63(0,44-0,89)$ & $1,13(0,91-1,42)$ & $1,17(0,98-1,41)$ & $1,03(0,89-1,20)$ \\
\hline \multicolumn{5}{|l|}{ Diabetes $^{1}$} \\
\hline Não & 1,00 & 1,00 & 1,00 & 1,00 \\
\hline $\operatorname{Sim}$ & $1,18(1,03-1,36)$ & $1,10(0,95-1,27)$ & $1,19(1,06-1,34)$ & $0,97(0,88-1,08)$ \\
\hline \multicolumn{5}{|l|}{ Hipertensão arterial $^{2}$} \\
\hline Não & 1,00 & 1,00 & 1,00 & 1,00 \\
\hline Sim & $1,08(0,97-1,21)$ & $1,01(0,90-1,13)$ & $1,00(0,90-1,10)$ & $1,05(0,98-1,13)$ \\
\hline \multicolumn{5}{|l|}{ Doença cardiovascular ${ }^{3}$} \\
\hline Não & 1,00 & 1,00 & 1,00 & 1,00 \\
\hline Sim & $0,99(0,86-1,15)$ & $0,96(0,82-1,12)$ & $0,92(0,80-1,06)$ & $1,06(0,97-1,15)$ \\
\hline \multicolumn{5}{|c|}{ Comprometimento cognitivo $^{4}$} \\
\hline Não & 1,00 & 1,00 & 1,00 & 1,00 \\
\hline Sim & $1,00(0,88-1,15)$ & $1,13(0,99-1,28)$ & $0,97(0,86-1,09)$ & $0,99(0,91-1,08)$ \\
\hline \multicolumn{5}{|l|}{ Limitação funcional $^{5}$} \\
\hline Não & 1,00 & 1,00 & 1,00 & 1,00 \\
\hline Sim & $1,27(1,11-1,44)$ & $0,93(0,80-1,10)$ & $1,07(0,94-1,21)$ & $0,89(0,80-0,99)$ \\
\hline \multicolumn{5}{|l|}{ Interleucina 6} \\
\hline$<1,08 \mathrm{pg} / \mathrm{mL}$ & 1,00 & 1,00 & 1,00 & 1,00 \\
\hline$\geq 1,08 \mathrm{pg} / \mathrm{mL}$ & $1,19(1,06-1,32)$ & $1,09(0,97-1,21)$ & $1,09(0,99-1,21)$ & $0,95(0,88-1,02)$ \\
\hline \multicolumn{5}{|l|}{ Proteína C-reativa } \\
\hline$<3,25 \mathrm{mg} / \mathrm{dL}$ & 1,00 & 1,00 & 1,00 & 1,00 \\
\hline$\geq 3,25 \mathrm{mg} / \mathrm{dL}$ & $1,20(1,07-1,33)$ & $1,02(0,92-1,14)$ & $1,03(0,93-1,13)$ & $0,95(0,88-1,01)$ \\
\hline \multicolumn{5}{|l|}{ Interleucina $1 \mathrm{~b}$} \\
\hline Não detectável & 1,00 & 1,00 & 1,00 & 1,00 \\
\hline$\geq 0,01 \mathrm{pg} / \mathrm{mL}$ & $0,95(0,83-1,09)$ & $0,97(0,84-1,10)$ & $0,97(0,86-1,10)$ & $0,88(0,80-0,97)$ \\
\hline \multicolumn{5}{|c|}{ Fator de necrose tumoral - TNF } \\
\hline Não detectável & 1,00 & 1,00 & 1,00 & 1,00 \\
\hline$\geq 0,02 \mathrm{pg} / \mathrm{mL}$ & $0,97(0,84-1,13)$ & $0,97(0,84-1,13)$ & $1,03(0,91-1,17)$ & $0,98(0,89-1,08)$ \\
\hline $\begin{array}{l}\text { RP (IC95\%): razões de prevalê } \\
\text { HSV: herpes simples (IgG }>16 \\
\text { U/mL). }{ }^{1} \text { Glicemia de jejum }>1 \\
\mathrm{mmHg} \text { e/ou tratamento; }{ }^{3} \mathrm{Ang}\end{array}$ & $\begin{array}{l}\text { s (intervalos de 95\% } \\
\text { lamydia pneumonia } \\
\text { o de medicamento; } \\
\text { lato de infarto do } \mathrm{n}\end{array}$ & $\begin{array}{l}\text { de confiança); CMV: } \\
\text { (IgG }>22 \mathrm{UR} / \mathrm{mL}) ; \mathrm{H} \\
\text { Pressão sistólica } \geq 14( \\
\text { iocárdio e/ou relato }\end{array}$ & $\begin{array}{l}\text { : citomegalovírus (Ig } \\
\text { HP: Helicobacter pylor } \\
0 \mathrm{mmHg} \text { e/ou pressã } \\
\text { de acidente vascular e }\end{array}$ & $\begin{array}{l}\mathrm{G}>399,5 \mathrm{U} / \mathrm{mL}) ; \\
i \text { (positivo }-\mathrm{IgG}>50 \\
\text { o diastólica } \geq 90 \\
\text { encefálico; }{ }^{4} \text { Escore } \\
\text {, deitar e levantar da }\end{array}$ \\
\hline
\end{tabular}


Tabela 3. Resultados da análise multivariada das associações entre citomegalovírus (CMV), herpes simples tipo 1 (HSV-1), C. pneumoniae (CP) e H. pylori (HP) e características sociodemográficas e comportamentais. Linha de base da Coorte de idosos de Bambuí.

\begin{tabular}{|c|c|c|c|c|}
\hline \multirow{2}{*}{ Variáveis } & \multicolumn{4}{|c|}{ RP ajustada (IC95\%) } \\
\hline & CMV & HSV & $\mathrm{CP}$ & HP \\
\hline \multicolumn{5}{|l|}{ Idade em anos } \\
\hline 60 a 74 & 1,00 & 1,00 & 1,00 & 1,00 \\
\hline 75 e mais & $1,13(1,01-1,28)$ & $1,02(0,90-1,16)$ & $1,15(1,04-1,28)$ & $0,94(0,86-1,02)$ \\
\hline \multicolumn{5}{|l|}{ Sexo } \\
\hline Homens & 1,00 & 1,00 & 1,00 & 1,00 \\
\hline Mulheres & $1,46(1,28-1,66)$ & $0,78(0,70-0,88)$ & $0,80(0,73-0,89)$ & $0,90(0,84-0,97)$ \\
\hline \multicolumn{5}{|l|}{ Escolaridade em anos } \\
\hline 4 ou mais & 1,00 & 1,00 & 1,00 & 1,00 \\
\hline$<4$ & $1,00(0,89-1,12)$ & $0,98(0,88-1,10)$ & $0,86(0,78-0,95)$ & $1,01(0,94-1,08)$ \\
\hline \multicolumn{5}{|l|}{ Tabagismo atual } \\
\hline Não & 1,00 & 1,00 & 1,00 & 1,00 \\
\hline Sim & $1,17(1,02-1,35)$ & $1,04(0,91-1,20)$ & $1,08(0,96-1,21)$ & $1,10(1,01-1,19)$ \\
\hline \multicolumn{5}{|c|}{ Consumo de álcool (doses/semana) } \\
\hline$<7$ & 1,00 & 1,00 & 1,00 & 1,00 \\
\hline 7 ou mais & $0,78(0,54-1,12)$ & $0,97(0,78-1,22)$ & $1,00(0,83-1,21)$ & $0,96(0,82-1,12)$ \\
\hline
\end{tabular}

RP (IC95\%): razões de prevalência ajustadas por todas as variáveis listadas na tabela (intervalos de 95\% de confiança); CMV: citomegalovírus (IgG>399,5 U/mL); HSV: herpes simples (IgG>160 UR/mL); CP: Chlamydia pneumoniae (IgG>22 UR/mL); HP: Helicobacter pylori (positivo - IgG $>50 \mathrm{U} / \mathrm{mL}$ ).

Fonte: Elaborado pelos autores.

a prevalência de infecção por $H$. pylori também foi menor entre as mulheres (RP: 0,90; IC95\%: 0,84-0,97), mas foi positivamente associada ao tabagismo atual (RP: 1,10; IC95\%: 1,01-1,19).

Os resultados da associação entre as quatro infecções pesquisadas e as variáveis relacionadas às condições de saúde, considerando o ajustamento por todas essas condições e pelos fatores sociodemográficos e comportamentais, estão descritos na Tabela 4. A prevalência de maiores títulos de anticorpos para CMV foi maior entre os idosos com diagnóstico de diabetes (RP: 1,15; IC95\%: 1,01-1,31), entre aqueles que reportaram limitações nas atividades de vida diária (RP: 1,20; IC95\%: 1,05-1,38) e no grupo com maiores níveis de IL-6 (RP: 1,14; IC95\%: 1,01-1,27). Por outro lado, o HSV-1 não apresentou associação significativa com nenhuma das condições de saúde avaliadas; a infecção por C. pneumoniae foi mais frequente entre os diabéticos (RP: 1,19; IC95\%: 1,06-1,33); e a infecção por $H$. pylori foi menos comum entre os idosos com níveis detectáveis de IL-1 $\beta$ (RP: 0,87; IC95\%: 0,79-0,97).

A análise de sensibilidade, excluindo-se os idosos com resultados inconclusivos para dosagens de anticorpos, mostrou que os resultados dos modelos ajustados mantiveram as associa- ções descritas nesse estudo, com a mesma direção e significância estatística, para todos os quatro patógenos investigados.

\section{Discussão}

As prevalências de infecções crônicas observadas entre os idosos de Bambuí podem ser consideradas relativamente elevadas, sugerindo maior vulnerabilidade aos eventos adversos associados às infecções ${ }^{5}$. Além disso, nossos resultados possibilitaram identificar o perfil epidemiológico que caracteriza essas infecções, revelando diferenças importantes entre os patógenos. De maneira geral, os idosos mais velhos apresentaram maiores títulos de anticorpos para o CMV e maior prevalência de infecção por C. pneumoniae; as mulheres apresentaram menor prevalência de CMV e maior prevalência das outras três infecções; os idosos com menor escolaridade tinham menor prevalência de C. pneumoniae e os fumantes atuais tinham maior prevalência para CMV e $H$. pylori. Em relação às condições de saúde, as infecções por CMV e C. pneumoniae foram mais frequentes entre os diabéticos; o CMV foi mais prevalente entre os idosos com incapacidade e 
Tabela 4. Resultados da análise multivariada das associações entre citomegalovírus (CMV), herpes simples tipo 1 (HSV-1), C. pneumoniae (CP) e H. pylori (HP) e características de saúde. Linha de base da Coorte de idosos de Bambuí.

\begin{tabular}{|c|c|c|c|c|}
\hline \multirow{2}{*}{ Variáveis } & \multicolumn{4}{|c|}{ RP ajustada (IC95\%) } \\
\hline & CMV & HSV & $\mathrm{CP}$ & HP \\
\hline \multicolumn{5}{|l|}{ Diabetes $^{1}$} \\
\hline Não & 1,00 & 1,00 & 1,00 & 1,00 \\
\hline Sim & $1,15(1,01-1,31)$ & $1,12(0,97-1,29)$ & $1,19(1,06-1,33)$ & $0,99(0,89-1,09)$ \\
\hline \multicolumn{5}{|l|}{ Hipertensão arterial $^{2}$} \\
\hline Não & 1,00 & 1,00 & 1,00 & 1,00 \\
\hline Sim & $1,02(0,91-1,14)$ & $1,03(0,92-1,16)$ & $1,02(0,93-1,13)$ & $1,07(0,99-1,15)$ \\
\hline \multicolumn{5}{|l|}{ Doença cardiovascular ${ }^{3}$} \\
\hline Não & 1,00 & 1,00 & 1,00 & 1,00 \\
\hline Sim & $0,93(0,81-1,08)$ & $0,97(0,83-1,13)$ & $0,94(0,82-1,08)$ & $1,07(0,98-1,17)$ \\
\hline \multicolumn{5}{|c|}{ Comprometimento cognitivo ${ }^{4}$} \\
\hline Não & 1,00 & 1,00 & 1,00 & 1,00 \\
\hline Sim & $0,99(0,86-1,15)$ & $1,12(0,98-1,29)$ & $0,94(0,83-1,07)$ & $0,98(0,89-1,07)$ \\
\hline \multicolumn{5}{|l|}{ Limitação funcional $^{5}$} \\
\hline Não & 1,00 & 1,00 & 1,00 & 1,00 \\
\hline Sim & $1,20(1,05-1,38)$ & $0,89(0,75-1,05)$ & $1,04(0,91-1,18)$ & $0,90(0,81-1,01)$ \\
\hline \multicolumn{5}{|l|}{ Interleucina 6} \\
\hline$<1,08 \mathrm{pg} / \mathrm{mL}$ & 1,00 & 1,00 & 1,00 & 1,00 \\
\hline$\geq 1,08 \mathrm{pg} / \mathrm{mL}$ & $1,14(1,01-1,27)$ & $1,08(0,96-1,21)$ & $1,07(0,97-1,18)$ & $0,96(0,89-1,03)$ \\
\hline \multicolumn{5}{|l|}{ Proteína C-reativa } \\
\hline$<3,25 \mathrm{mg} / \mathrm{dL}$ & 1,00 & 1,00 & 1,00 & 1,00 \\
\hline$\geq 3,25 \mathrm{mg} / \mathrm{dL}$ & $1,10(0,99-1,23)$ & $1,02(0,91-1,14)$ & $1,00(0,91-1,11)$ & $0,96(0,89-1,03)$ \\
\hline \multicolumn{5}{|l|}{ Interleucina $1 \mathrm{~b}$} \\
\hline Não detectável & 1,00 & 1,00 & 1,00 & 1,00 \\
\hline$\geq 0,01 \mathrm{pg} / \mathrm{mL}$ & $0,96(0,83-1,10)$ & $0,98(0,85-1,14)$ & $0,98(0,87-1,11)$ & $0,87(0,79-0,97)$ \\
\hline \multicolumn{5}{|c|}{ Fator de necrose tumoral - TNF } \\
\hline Não detectável & 1,00 & 1,00 & 1,00 & 1,00 \\
\hline$\geq 0,02 \mathrm{pg} / \mathrm{mL}$ & $0,94(0,80-1,10)$ & $0,97(0,83-1,14)$ & $1,03(0,90-1,17)$ & $1,05(0,95-1,17)$ \\
\hline $\begin{array}{l}\text { RP (IC95\%): razões de preval } \\
\text { de } 95 \% \text { de confiança); CMV: } \\
\text { pneumoniae (IgG>22 UR/mL) } \\
\text { medicamento; }{ }^{2} \text { Pressão sistóli } \\
\text { infarto do miocárdio e/ou rela } \\
\text { dificuldade para tomar banho } \\
\text { preparar refeições. }\end{array}$ & $\begin{array}{l}\text { or todas as variáveis li } \\
\text { IgG }>399,5 \mathrm{U} / \mathrm{mL}) ; \mathrm{H} \\
\\
\text { /ou presi (positivo - Ig } \\
\text { sscular encefálicosólica }{ }^{4} \mathrm{Esc} \\
\text { tar-se, deitar e levant }\end{array}$ & $\begin{array}{l}\text { stadas na tabela, além } \\
\text { SV: herpes simples (Ig } \\
\text { G>50 U/mL). }{ }^{1} \text { Glicem } \\
\geq 90 \mathrm{mmHg} \text { e/ou trat } \\
\text { ore do Mini-exame d } \\
\text { ar da cama, lidar com }\end{array}$ & $\begin{array}{l}\text { de idade, sexo e escc } \\
\mathrm{G}>160 \mathrm{UR} / \mathrm{mL} \text { ); } \mathrm{CP} \\
\text { ia de jejum }>126 \mathrm{mg} \\
\text { amento; }{ }^{3} \text { Angina }(\mathrm{pr} \\
\text { o estado mental }<22 \\
\text { finanças, fazer tarefa }\end{array}$ & $\begin{array}{l}\text { laridade (intervalos } \\
\text { : Chlamydia } \\
\text { /dL e/ou uso de } \\
\text { otocolo), relato de } \\
\text {; }{ }^{5} \text { Relato de alguma } \\
\text { s domésticas ou }\end{array}$ \\
\hline
\end{tabular}

Fonte: Elaborado pelos autores.

naqueles com maiores níveis de IL-6 e a infecção por H. pylori foi menos frequente entre os idosos com níveis detectáveis de IL-1 $\beta$.

As prevalências para infecção por CMV e HSV-1 apresentam ampla variação entre populações, podendo atingir níveis elevados, como observado na presente análise (99,4\% e 96,7\%, respectivamente), sobretudo em regiões menos desenvolvidas ${ }^{6,7}$. A prevalência de infecção pelo CMV em Bambuí foi semelhante ao observado entre adultos mexicanos $(97,0 \%)^{24}$ e superior ao observado entre homens $(64,7 \%)$ e mulheres
(77,6\%) com idades entre 70 e 79 anos da Alemanha ${ }^{10}$. Já a infecção pelo HSV-1 atingiu valores elevados entre adultos africanos (>91\%) e entre americanos com 70 anos ou mais $(90 \%)^{7}$, de forma semelhante ao descrito na presente investigação. Nossos resultados acrescentam por mostrar prevalência elevada de títulos de anticorpos contra esses patógenos em uma população idosa brasileira, caracterizando um processo crônico de infecção nesse grupo.

A prevalência de infecção por C. pneumoniae $(56,0 \%)$ esteve entre os valores observados 
na literatura, que variaram de $45,0 \%{ }^{25}$ a $70,9 \%{ }^{3}$ nos Estados Unidos, chegando a 75,0\% entre adultos italianos ${ }^{8}$. Já a prevalência de $H$. pylori (70,5\%) apresentou valor semelhante ao reportado em meta-análise para a população brasileira $(71,2 \%)$, inferior ao observado na África $(79,1 \%)$ e muito superior às regiões de maior renda, como Europa $(47,0 \%)$ e América do Norte $(37,1 \%)^{9}$. Portanto, tal como observado para as infecções virais, os resultados ressaltaram a elevada prevalência de infecções bacterianas nessa população, contribuindo para o conhecimento da epidemiologia das infecções entre idosos.

$\mathrm{O}$ aumento da prevalência de infecção por CMV com a idade já foi observado em outras populações ${ }^{6,10,13,26}$, apresentando também aumento da carga viral, mesmo após os 60 anos de idade ${ }^{27}$, estando em consonância com nossos resultados, podendo representar uma exposição passada e cumulativa. De forma também semelhante a outros estudos, a infecção por CMV foi mais comum entre as mulheres de Bambuí, o que pode ser atribuído ao papel cultural de cuidar de crianças, seja em casa ou nas escolas, estando mais expostas à transmissão desse vírus ao longo da vida ${ }^{6,10}$.

A prevalência da infecção pelo HSV-1 tende a aumentar com a idade ${ }^{11,24}$, mas a maioria dos estudos foi conduzida em populações adultas, crianças ou gestantes $^{7,28}$. Nossos resultados não evidenciaram aumento da prevalência a partir dos 60 anos, o que parece ser coerente com a hipótese de estabilização da prevalência entre os mais velhos ${ }^{7}$. Em relação à comparação entre os sexos, a prevalência de maiores níveis de anticorpos para HSV-1 foi significativamente menor entre as mulheres idosas de Bambuí, corroborando a hipótese levantada em estudo de revisão, de que há redução dessa prevalência entre as mulheres com o envelhecimento ${ }^{7}$. A distribuição da infecção por C. pneumoniae apresenta variações em relação à idade e sexo ${ }^{8,29}$, mas de forma semelhante ao observado em Bambuí, essa prevalência entre adultos italianos aumentou com a idade e foi maior entre homens ${ }^{8}$. Já a prevalência de infecção por $H$. pylori é consistentemente maior entre os homens ${ }^{25,30,31}$ e não apresenta grande variação com idade a partir dos $60 \operatorname{anos}^{30,32}$, tal como observado em Bambuí.

Alguns resultados obtidos em países de alta renda mostram que as prevalências de infecções crônicas foram mais frequentes nos grupos de pior nível socioeconômico ${ }^{11,12,28,33,34}$, mas esses estudos incluíram, predominantemente, populações adultas. Por outro lado, estudo conduzido no México não evidenciou associação entre escolaridade e as infecções por HSV-1 e CMV ${ }^{24}$, bem como estudos conduzidos no Irã para $H$. pylori $i^{35}$ e na Itália para C. pneumoniae ${ }^{8}$. Esses resultados podem sugerir menor impacto do nível socioeconômico nessas infecções em países de baixa e média renda ou menor efeito da posição econômica em populações idosas, sendo coerente ao que foi descrito no presente estudo. Pode-se supor ainda que a situação socioeconômica na infância pode ser mais importante para a determinação dessas infecções do que a situação avaliada na fase adulta, sobretudo a partir dos $60 \operatorname{anos}^{36}$. Em relação à C. pneumoniae, a condição socioeconômica e escolaridade podem não ser relevantes para a prevalência dessa infecção ${ }^{8}$, mas entre idosos de Bambuí o grupo com menor escolaridade apresentou menor prevalência de infecção por $C$. pneumoniae, mesmo após ajuste por idade, sexo e tabagismo, diferindo das evidências reportadas até o presente momento.

A não associação entre as infecções avaliadas no presente estudo e o consumo de álcool é coerente com resultados observados em outras populações ${ }^{24,37}$, embora já tenha sido descrita associação negativa entre o consumo de álcool e a infecção por $H$. pylori ${ }^{12}$, o que não foi observado no presente estudo. Sobre o hábito de fumar, a prevalência de maior titulação de anticorpos para CMV e de infecção pelo $H$. pylori foi maior entre os tabagistas atuais em Bambuí, tal como observado na Inglaterra para $\mathrm{CMV}^{11}$ e na Alemanha para $\mathrm{CMV}^{10,34}$ e $H$. pylori ${ }^{12}$. Ainda não está claro se essa associação ocorre pela influência do tabaco no sistema imunológico, favorecendo a infecção ${ }^{38}$ ou se o hábito de fumar seria um proxi para outros comportamentos não saudáveis ou pior nível socioeconômico ${ }^{10,34}$. De toda forma, nossos resultados reforçam que o tabagismo parece apresentar uma associação mais consistente com infecções crônicas em comparação ao consumo de álcool, mesmo em populações idosas, sobretudo para CMV e H. pylori.

A associação entre CMV e diabetes permaneceu significativa mesmo após ajuste pelos confundidores, confirmando resultados obtidos em outras populações ${ }^{4,13,37}$. A hipótese de que essa relação pudesse ser explicada pela idade e outros fatores de confusão ${ }^{13}$ não deve ter ocorrido no presente estudo, considerando a persistência da significância estatística na análise múltipla. De forma semelhante, a associação entre diabetes e C. pneumoniae está em consonância com resultado anterior ${ }^{39}$, podendo ser explicada pela influência dessa infecção nas células beta do pân- 
creas, aumentando a chance de ocorrência do diabetes $^{39}$. Em relação ao HSV-1, os resultados de Bambuí são consonantes com outras populações ${ }^{2,11}$, reforçando a inexistência de relação entre essa infecção e o diabetes. Por outro lado, a associação entre infecção por $H$. pylori e diabetes ainda é controversa na literatura ${ }^{40}$, embora em meta-análise recente foi observada associação significativa entre essas condições ${ }^{41}$, de forma oposta ao observado entre idosos de Bambuí.

Ainda que possa haver mecanismos que justifiquem a associação entre algumas infecções crônicas e as doenças cardiovasculares, os resultados são controversos entre as populações ${ }^{15}$. Em Bambuí, nenhuma das quatro infecções avaliadas apresentou associação significativa com hipertensão e com doenças cardiovasculares, reforçando resultados anteriores ${ }^{25,42}$. Ressalta-se a importância da inflamação, além da condição socioeconômica e hábito de fumar, como possíveis mecanismos que explicariam essas associações ${ }^{15,40}$, mas no presente estudo essas associações não foram observadas mesmo na análise não ajustada.

A limitação funcional não esteve associada a infecções por C. pneumoniae, H. pylori e HSV-1, de forma coerente com outros estudos ${ }^{2,16,43}$, enquanto o CMV parece exercer papel importante na ocorrência de limitações físicas entre idosos, como já demonstrado anteriormente $e^{2,14,44}$. Embora alguns estudos não evidenciaram significância estatística após ajustamento por fatores de confusão $0^{4,43}$, nossos resultados reforçam essa associação, pois ela se manteve mesmo após ajustamento por diversos confundidores, incluindo marcadores do perfil inflamatório, que parece ter papel importante nessa associação ${ }^{43,44}$. Por sua vez, a associação entre cognição e algumas infecções crônicas já foi descrita anteriormente ${ }^{1}$, com maior influência das infecções virais ${ }^{45}$, mas esse resultado não foi observado entre idosos de Bambuí, demonstrando que o comprometimento cognitivo ocorre de forma independente das infecções investigadas.

De maneira geral, infecções crônicas têm sido associadas com maiores níveis de marcadores inflamatórios entre idosos, com destaque para o CMV, contribuindo para o processo de imunosenescência ${ }^{46,47}$. No presente estudo a IL-6 apresentou associação com CMV após ajustamento pelos confundidores, estando em consonância com o papel desse patógeno no processo conhecido como inflamm-aging. Sugere-se que a presença dessa infecção e sua reativação está relacionada com redução do repertório de células $T$ CD8+CD28-, redução do número de células $\mathrm{B}$ e aumento dos níveis de IL-6 no plasma ${ }^{46}$, justificando os resultados observados em Bambuí. Já a infecção por H. pylori pode se apresentar associada com aumento dos marcadores inflamatórios ${ }^{48}$, mas em outras populações essa associação não foi observada $^{31,49}$. No entanto, já foi verificado que a presença dessa infecção pode suprimir a produção de citocinas pró-inflamatórias pela redução do estresse oxidativo ${ }^{40}$, o que é coerente com o resultado observado entre idosos de Bambuí, com menor produção de IL-1 $\beta$ entre os infectados.

A principal limitação deste trabalho é o delineamento seccional, que não permite estabelecer relações temporais entre as variáveis pesquisadas, embora o objetivo tenha sido detectar o perfil dos idosos com infecções crônicas em um estudo de base populacional. Além disso, a dosagem de anticorpos não permite avaliar se a infecção é recente ou se pode se relacionar a uma reativação dos patógenos, mostrando o nível de IgG no momento da coleta dos dados. O presente estudo inclui 1.320 idosos que possuíam informações completas para todas as variáveis, entre os 1.606 que participaram do inquérito. $\mathrm{O}$ grupo de idosos não incluídos nessa análise era significativamente mais velho e apresentava maior proporção de mulheres, com menor escolaridade e maiores prevalências de comprometimento cognitivo e limitação funcional. As demais variáveis não apresentaram diferenças significativas. Portanto, as prevalências das infecções crônicas podem ter sido subestimadas e a magnitude das associações reduzidas. No entanto, ressalta-se que a amostra investigada era suficiente para detectar uma diferença mínima de $8 \%$ entre os grupos, mantendose um poder maior ou igual a $80 \%$.

Por outro lado, a análise foi conduzida em um estudo de base populacional, com informações coletadas por instrumentos padronizados e examinadores treinados, buscando assegurar a qualidade das informações coletadas. Além disso, o estudo incluiu quatro infecções, que apresentam potenciais impactos para o envelhecimento, e os resultados podem contribuir para o conhecimento acerca do perfil dos idosos com infecções crônicas residentes em um país latino-americano, considerando a escassez de estudos nessa região.

Em resumo, os resultados do presente estudo mostraram elevada prevalência para as quatro infecções estudadas, além de demonstrar que os fatores associados a essas infecções se apresentaram de forma diferente para cada patógeno, permitindo caracterizar os grupos mais vulneráveis. Ainda assim, e em consonância com a falta de consistência observada na literatura sobre a as- 
sociação entre os patógenos e os eventos adversos, fica evidente a necessidade de outros estudos, envolvendo populações residentes em diferentes regiões e incluindo diferentes faixas etárias, que possam contribuir para o conhecimento da epidemiologia das infecções crônicas, com destaque para seu efeito entre idosos e para o papel da inflamação nesse processo.

\section{Colaboradores}

SV Peixoto participou da concepção, delineamento, análise e interpretação dos dados, redação do manuscrito e revisão e aprovação da versão final. KCL Torres participou da interpretação dos resultados, revisão crítica do conteúdo intelectual e aprovação da versão final. A Teixeira-Carvalho participou da interpretação dos resultados, revisão crítica do conteúdo intelectual e aprovação da versão final. OA Martins-Filho participou da interpretação dos resultados, revisão crítica do conteúdo intelectual e aprovação da versão final. MF Lima-Costa participou da concepção e delineamento do estudo, análise e interpretação dos dados, revisão crítica e aprovação da versão final.

\section{Agradecimento}

SV Peixoto, A Teixeira-Carvalho, OA Martins-Filho e MF Lima-Costa são bolsistas de produtividade do Conselho Nacional de Desenvolvimento Científico e Tecnológico (CNPq).

\section{Financiamento}

Instituto René Rachou (Fiocruz Minas), Fundação de Amparo à Pesquisa do Estado de Minas Gerais (FAPEMIG) e Conselho Nacional de Desenvolvimento Científico e Tecnológico (CNPq). 


\section{Referências}

1. Aiello AE, Haan M, Blythe L, Moore K, Gonzalez JM, Jagust W. The influence of latent viral infection on rate of cognitive decline over 4 years. J Am Geriatr Soc 2006; 54(7):1046-1054.

2. Haeseker MB, Pijpers E, Dukers-Muijers NHTM, Nelemans P, Hoebe CJ, Bruggeman CA, Verbon A, Goossens VJ. Association of cytomegalovirus and other pathogens with frailty and diabetes mellitus, but not with cardiovascular disease and mortality in psychogeriatric patients; a prospective cohort study. Immun Ageing 2013; 10(1):30.

3. Szklo M, Ding J, Tsai MY, Cushman M, Polak JF, Lima J, Barr RG, Sharrett AR. Individual pathogens, pathogen burden, and markers of subclinical atherosclerosis: the Multi-Ethnic Study of Atherosclerosis. J Carsiovasc Med (Hagerstown) 2009; 10(10):747-751.

4. Wang GC, Kao WHL, Murakami P, Xue QL, Chiou RB, Detrick B, McDyer JF, Semba RD, Casolaro V, Walston JD, Fried LP. Cytomegalovirus Infection and the Risk of Mortality and Frailty in Older Women: A Prospective Observational Cohort Study. Am J Epidemiol 2010; 171(10):1144-1152.

5. Gavazzi G, Krause K. Ageing and infection. Lancet Infect Dis 2002; 2(11):659-666.

6. Cannon MJ, Schmid DS, Hyde TB. Review of cytomegalovirus seroprevalence and demographic characteristics associated with infection. Rev Med Virol 2010; 20(4):202-213.

7. Smith JS, Robinson NJ. Age-specific prevalence of infection with herpes simplex virus types 2 and 1: a global review. J Infect Dis 2002; 186(Supl. 1):S3-S28.

8. Blanc P, Corsi AM, Gabbuti A, Peduzzi C, Meacci F, Olivieri F, Lauretani F, Francesco M, Ferrucci L. Chlamydia pneumoniae seropositivity and cardiovascular risk factors: The InCHIANTI Study. J Am Geriatr Soc 2004; 52(10):1626-1631.

9. Hooi JKY, Lai WY, Ng WK, Suen MMY, Underwood FE, Tanyingoh D, Malfertheiner P, Graham DY, Wong VWS, Wu JCY, Chan FKL, Sung JJY, Kaplan GG, Ng SC. Global Prevalence of Helicobacter pylori Infection: Systematic Review and Meta-Analysis. Gastroenterology 2017; 153(2):420-429.

10. Lachmann R, Loenenbach A, Waterboer $\mathrm{T}$, Brenner N, Pawlita M, Michel A, Thamm M, Poethko-Müller C, Wichmann O, Wiese-Posselt M. Cytomegalovirus (CMV) seroprevalence in the adult population of Germany. PLoS One 2018; 13(7):e0200267.

11. Forbes H, Warne B, Doelken L, Brenner N, Waterboer T, Luben R, Wareham NJ, Warren-Gash C, Gkrania -Klotsas E. Risk factors for herpes simplex virus type1 infection and reactivation: Cross-sectional studies among EPIC-Norfolk participants. PLoS One 2019; 14(5):e0215553.

12. Wawro N, Amann U, Butt J5, Meisinger C, Akmatov MK, Pessler F, Peters A, Rathmann W, Kääb S, Waterboer T, Linseisen J. Helicobacter pylori Seropositivity: Prevalence, Associations, and the Impact on Incident Metabolic Diseases/Risk Factors in the Population -Based KORA Study. Front Public Health 2019; 7:96.
13. Schmidt L, Nelson HH, Thyagarajan B, Hunter-Schlichting D, Pankow JS, Capistrant B, Prizment AE. Association between cytomegalovirus seropositivity and Type 2 diabetes is explained by age and other demographic characteristics: the National Health and Nutrition Examination Survey. Diabet Med 2018; 35(12):1722-1726.

14. Thomasini RL, Pereira DS, Pereira FSM, Mateo EC, Mota TN, Guimarães GG, Pereira LSM, Lima CX, Teixeira MM, Teixeira AL Junior. Aged-associated cytomegalovirus and Epstein-Barr virus reactivation and cytomegalovirus relationship with the frailty syndrome in older women. PLoS One 2017; 12(7):e0180841.

15. Rezaee-Zavareh MS, Tohidi M, Sabouri A, RamezaniBinabaj M, Sadeghi-Ghahrodi M, Einollahi B. Infectious and coronary artery disease. ARYA Atheroscler 2016; 12(1):41-49.

16. Araújo Carvalho AC, Tavares Mendes ML, Santos VS, Tanajura DM, Prado Nunes MA, Martins-Filho PRS. Association between human herpes virus seropositivity and frailty in the elderly: A systematic review and meta-analysis. Ageing Res Rev 2018; 48:145-152.

17. Roberts ET, Haan MN, Dowd JB, Aiello AE. Cytomegalovirus Antibody Levels, Inflammation, and Mortality Among Elderly Latinos Over 9 Years of Follow-up. Am J Epidemiol 2010; 172(4):363-371.

18. Franceschi C, Campisi J. Chronic inflammation (inflammaging) and its potential contribution to age-associated diseases. J Gerontol A Biol Sci Med Sci 2014; 69(Supl. 1):S4-S9.

19. Lima-Costa MF, Firmo JO, Uchoa E. Cohort profile: the Bambuí (Brazil) Cohort Study of Ageing. Int J Epidemiol 2011; 40(4):862-867.

20. Moos RH, Schutte KK, Brennan PL, Moos BS. Older adults' alcohol consumption and late-life drinking problems: a 20- year perspective. Addiction 2009; 104(8):1293-1302.

21. Plan and operation of the Third National Health and Nutrition Examination Survey, 1988-94. Series 1: programs and collection procedures. Vital Health Stat 1 1994; 32:1-407.

22. Rose G. The diagnosis of ischaemic heart pain and intermittent claudication in field surveys. Bull WHO 1962; 27:645-658.

23. Castro-Costa E, Fuzikawa C, Uchoa E, Firmo JO, Lima-Costa MF. Norms for the mini-mental state examination: adjustment of the cut-off point in population-based studies (evidences from the Bambuí health aging study). Arq Neuro-Psiquiatr 2008; 66(3a):524528.

24. Conde-Glez C, Lazcano-Ponce E, Rojas R, DeAntonio R, Romano-Mazzotti L, Cervantes Y, Ortega-Barria E. Seroprevalences of varicella-zoster virus, herpes simplex virus and cytomegalovirus in a cross-sectional study in Mexico. Vaccine 2013; 31(44):5067-5074.

25. Haider AW, Wilson PW, Larson MG, Evans JC, Michelson EL, Wolf PA, O’Donnell CJ, Levy D. The association of seropositivity to Helicobacter pylori, Chlamydia pneumoniae, and cytomegalovirus with risk of cardiovascular disease: a prospective study. $J$ Am Coll Cardiol 2002; 40(8):1408-1413. 
26. Olsson J, Kok E, Adolfsson R, Lövheim H, Elgh F Herpes virus seroepidemiology in the adult Swedish population. Immun Ageing 2017; 14:10.

27. Parry HM, Zuo J, Frumento G, Mirajkar N, Inman C, Edwards E, Griffiths M, Pratt G, Moss P. Cytomegalovirus viral load within blood increases markedly in healthy people over the age of 70 years. Immun Ageing 2016; 13:1.

28. Woestenberg PJ, Tjhie JH, de Melker HE, van der Klis FR, van Bergen JE, van der Sande MA, van Benthem BH. Herpes simplex virus type 1 and type 2 in the Netherlands: seroprevalence, risk factors and changes during a 12-year period. BMC Infect Dis 2016; 16:364.

29. Cui J, Yan W, Xie H, Xu S, Wang Q, Zhang W, Ni A. A retrospective seroepidemiologic survey of Chlamydia pneumoniae infection in patients in Beijing between 2008 and 2017. PLoS One 2018; 13(11):e0206995.

30. Lim SH, Kwon JW, Kim N, Kim GH, Kang JM, Park MJ, Yim JY, Kim HU, Baik GH, Seo GS, Shin JE, Joo YE, Kim JS, Jung HC. Prevalence and risk factors of Helicobacter pylori infection in Korea: nationwide multicenter study over 13 years. BMC Gastroenterol 2013; 13:104.

31. Kim TJ, Pyo JH, Lee H, Baek SY, Ahn SH, Min YW, Min BH, Lee JH, Son HJ, Rhee PL, Kim JJ. Lack of Association between Helicobacter pylori Infection and Various Markers of Systemic Inflammation in Asymptomatic Adults. Korean J Gastroenterol 2018; 72(1):21-27.

32. Shi R, Xu S, Zhang H, Ding Y, Sun G, Huang X, Chen X, Li X, Yan Z, Zhang G. Prevalence and risk factors for Helicobacter pylori infection in Chinese populations. Helicobacter 2008; 13(2):157-165.

33. Bradley H, Markowitz LE, Gibson T, McQuillan GM. Seroprevalence of herpes simplex virus types 1 and 2-United States, 1999-2010. J Infect Dis 2014; 209(3):325-333.

34. Korr G, Thamm M, Czogiel I, Poethko-Mueller C, Bremer V, Jansen K. Decreasing seroprevalence of herpes simplex virus type 1 and type 2 in Germany leaves many people susceptible to genital infection: time to raise awareness and enhance control. BMC Infect Dis 2017; 17(1):471.

35. Maleki I, Mohammadpour M, Zarrinpour N, Khabazi M, Mohammadpour RA. Prevalence of Helicobacter pylori infection in Sari Northern Iran; a population based study. Gastroenterol Hepatol Bed Bench 2019; 12(1):31-37.

36. Meier HCS, Haan MN, Mendes de Leon CF, Simanek AM, Dowd JB, Aiello AE. Early life socioeconomic position and immune response to persistent infections among elderly Latinos. Soc Sci Med 2016; 166:77-85.

37. Chen S, de Craen AJ, Raz Y, Derhovanessian E, Vossen AC, Westendorp RG, Pawelec G, Maier AB. Cytomegalovirus seropositivity is associated with glucose regulation in the oldest old. Results from the Leiden 85-plus Study. Immun Ageing 2012; 9(1):18.

38. Qiu F, Liang CL, Liu H, Zeng YQ, Hou S, Huang S, Lai $\mathrm{X}$, Dai Z. Impacts of cigarette smoking on immune responsiveness: Up and down or upside down? Oncotarget 2017; 8(1):268-284.
39. Rodriguez AR, Plascencia-Villa G, Witt CM, Yu JJ, José-Yacamán M, Chambers JP, Perry G, Guentzel MN, Arulanandam BP. Chlamydia pneumoniae promotes dysfunction of pancreatic beta cells. Cell Immunol 2015; 295(2):83-91.

40. Bravo D, Hoare A, Soto C, Valenzuela MA, Quest AF. Helicobacter pylori in human health and disease: Mechanisms for local gastric and systemic effects. World J Gastroenterol 2018; 24(28):3071-3089.

41. Li JZ, Li JY, Wu TF, Xu JH, Huang CZ, Cheng D, Chen QK, Yu T. Helicobacter pylori Infection Is Associated with Type 2 Diabetes, Not Type 1 Diabetes: An Updated Meta-Analysis. Gastroenterol Res Pract 2017; 5715403

42. Patel P, Mendall MA, Carrington D, Strachan DP, Leatham E, Molineaux N, Levy J, Blakeston C, Seymour CA, Camm AJ. Association of Helicobacter pylori and Chlamydia pneumoniae infections with coronary heart disease and cardiovascular risk factors. BMJ 1995; 311(7007):711-714.

43. Aiello AE, Haan MN, Pierce CM, Simanek AM, Liang J. Persistent infection, inflammation, and functional impairment in older Latinos. J Gerontol A Biol Sci Med Sci 2008; 63(6):610-618.

44. Schmaltz HN, Fried LP, Xue Q, Walston J, Leng SX, Semba RD. Chronic Cytomegalovirus Infection and Inflammation Are Associated with Prevalent Frailty in Community-Dwelling Older Women. J Am Geriatr Soc 2005; 53(5):747-754.

45. Itzhaki RF. Herpes simplex virus type 1 and Alzheimer's disease: possible mechanisms and signposts. FASEB J 2017; 31(8):3216-3226.

46. Bauer ME, Fuente ML. The role of oxidative and inflammatory stress and persistent viral infections in immunosenescence. Mech Ageing Dev 2016; 158:2737.

47. Pawelec G. Immunosenenescence: role of cytomegalovirus. Exp Gerontol 2014; 54:1-5.

48. Jackson L, Britton J, Lewis SA, McKeever TM, Atherton J, Fullerton D, Fogarty AW. A population-based epidemiologic study of Helicobacter pylori infection and its association with systemic inflammation. Helicobacter 2009; 14(5):108-113.

49. Chen ST, Ni YH, Li CC, Liu SH. Hepcidin correlates with interleukin- $1 \beta$ and interleukin- 6 but not iron deficiency in children with Helicobacter pylori infection. Pediatr Neonatol 2018; 59(6):611-617.

Artigo apresentado em 24/10/2019

Aprovado em 21/01/2020

Versão final apresentada em 23/01/2020

Editores-chefes: Romeu Gomes, Antônio Augusto Moura da Silva 
